Research Paper

\title{
Polyclonal Rabbit Anti-Cancer-Associated Fibroblasts Globulins Induce Cancer Cells Apoptosis and Inhibit Tumor Growth
}

\author{
Xiuying $\mathrm{Li}^{1}{ }^{\bowtie}$, Fengchang Huang ${ }^{2}$, Xiaoyu $\mathrm{Xu}^{3}$, Shuenqin $\mathrm{Hu}^{4}$ \\ 1. Medical Faculty, Kunming University of Science and Technology, Kunming, China \\ 2. Department of Oncology, First Affiliated Hospital of Kunming Medical University, Kunming, China \\ 3. College of Pharmaceutical Sciences \& College of Chinese Medicine, Southwest University, Chongqing 400715, China \\ 4. Department of Gynecology and Obstetrics, the First Affiliated Hospital of Kunming, Medical University, Kunming, China \\ $\triangle$ Corresponding author: XY LI, Medical Faculty of Kunming University of Science and Technology, Kunming, Yunnan, 650093, China. E-mail: \\ xiuyingli2013@kmust.edu.cn; Phone: 86-871-65920653; Fax: 86-871-65920653. \\ (c) Ivyspring International Publisher. This is an open access article distributed under the terms of the Creative Commons Attribution (CC BY-NC) license \\ (https://creativecommons.org/licenses/by-nc/4.0/). See http://ivyspring.com/terms for full terms and conditions.
}

Received: 2018.04.06; Accepted: 2018.08.18; Published: 2018.09.07

\begin{abstract}
Cancer-associated fibroblasts (CAFs) constitute a major component of the tumor microenvironment. CAFs regulated the growth and development, invasion and metastasis of primary tumors, as well as response to treatment. Recent studies indicated that monoclonal antibody therapies had limited success, thus more effective polyclonal antibodies (Poly Abs) is urgently needed. Poly Abs is a possible alternative because they target multiple antigens simultaneously. In this report, we prepared Poly Abs by immunizing rabbits with the bFGF-activated fibroblasts. The Poly Abs inhibited the cancer cells proliferation as revealed by MTT analysis. The Poly Abs induced apoptosis as indicated by flow cytometric analysis, and microscopic observation of apoptotic changes in morphology. Compared with the control IgG, Poly Abs significantly inhibited tumor cells migration as indicated by wound healing and transwell analysis in vitro, and lung metastasis analysis in vivo. Serial intravenous injections of Poly Abs inhibited tumor growth in mice bearing murine CT26 colon carcinoma. Ki67 analysis indicated that Poly Abs significantly inhibited tumor cells proliferation, as compared to control lg G treatments. Our findings suggested that Poly Abs was an effective agent for apoptosis induction, migration and metastasis inhibition. The Poly Abs may be useful as a safe anticancer agent for cancer immunotherapy in the future.
\end{abstract}

Key words: polyclonal antibodies; immunotherapy; cancer-associated fibroblasts; apoptosis

\section{Introduction}

Mutations may evoke neoplastic phenotypes in normal cells. These stepwise accumulations of mutation alone generally do not result in cancer formation. The interaction and crosstalk between incipient cancer cells and the supporting cells which form the tumor microenvironment (TME) endow incipient cancer cells to acquire the traits that enable them to become tumorigenic and ultimately malignant. This indicates that TME plays a critical role in cancer development $[1,2]$. The tumor tissue consists of cancer cells and tumor-associated host cells. Tumor cells recruit non-malignant cells to their vicinity, and interact with these surrounding stromal cells. Each non-malignant cell type exerts it's function in the tumor microenvironment in order to regulate the initiation, progression, metastasis and chemoresistance of tumor cells [1,2]. The TME also played key role in help the tumor cells to escape from the immune system. Emerging evidence suggested that the TME can shape anti-tumor immunity and responsiveness to immunotherapy. Many efforts have been devoted to established and continuously updated that the TME provides targets for efficient immunotherapy, such as checkpoint blockade [3], bispecifc antibodies [4], and chimeric antigen receptor-modified T- (CAR T) cell-based therapy [5].

Cancer-associated fibroblasts (CAFs) are the principal component in the tumor microenvironment. CAFs commonly display myofibroblastic characteristics, including a prominent contractile ability and 
expression of a smooth muscle actin (a-SMA) [6]. It has been well established that CAFs play an important role in the pathogenesis and progression of tumor. CAFs are crucial in modulating the delivery of therapeutic agents via secreting CAF-specific proteins, cytokines, growth factors, and producing an extracellular matrix (ECM) [7-9]. Quiescent fibroblasts become activated in tumor microenvironment, and the activated fibroblasts are key regulators of the paracrine signaling between stromal and cancer cells $[6,10]$. Our previous study indicated that basic fibroblast growth factor (bFGF) could activate normal fibroblasts, and these activated fibroblasts had analogous properties with CAFs [11].

Antibody has been used for therapy in various forms for over a century [12]. The monoclonal antibody provides the advantage of specificity, but lacks validity in the treatment of diseases caused by complex target antigens [13,14], and problems with significant relapse rates and drug resistance remain. Bispecific antibodies (BsAbs) also have a potential drawback because they target only two different tumor-associated antigens (TAAs). Thus, it is believed that polyclonal antibodies (Poly Abs) have the ability to tackle complex target antigens, and reduced susceptibility to immune escape of cancer cells [15]. Moreover, the vast majority of previous and ongoing vaccine trials have been developed to target gene products associated with cancer cells themselves. Unfortunately, cancer cells are etiologically heterogeneous and express numerous different cellular surface antigens. Thus, targeting multiple antigens at the same time by efficient polyclonal antibodies is a potential alternative strategy $[16,17]$.

CAFs are regarded as an ideal and appropriate candidate for cancer immunotherapy. The antibodies targeting the CAFs are under development. In this study, we produced polyclonal rabbit anti-CAFs antibodies (Poly Abs) by immunizing rabbits with the bFGF-activated fibroblasts. Poly Abs could inhibit proliferation and induce apoptosis of cancer cells. Furthermore, they significantly inhibited tumor growth and tumor cells migration. These results indicated that Poly Abs may simultaneously act on multiple targets on cancer cells. Importantly, such strategy might provide new direction for tumor immunotherapy based on the whole characteristics of tumors.

\section{Materials and Methods}

\section{Animals and cell lines}

New Zealand White Rabbits and 6-8 week old $\mathrm{BALB} / \mathrm{c}$ and C57 mice were purchased from the Hunan Slake Jingda Experimental Animal Co., Ltd (China). The mice were housed for at least 1 week before experiment. All the animal experiments were performed in accordance with China's Guidelines for Care and Use of Laboratory Animals. In this study, the BALB/c mouse embryonic fibroblast (3T3), CT26 colon carcinoma (CT26), LL/2 Lewis lung carcinoma (LL/2) and B16 melanoma (B16) cells obtained from the Chinese Academy of Sciences Kunming Cell Bank (Kunming, China). Cells were cultured in Dulbecco's modified Eagle's medium (DMEM, BI, Israel) containing $10 \%$ heat-inactivated fetal bovine serum (FBS, BI, Israel) with 100 units $/ \mathrm{ml}$ penicillin, and 100 units/ml streptomycin, in a humidified $5 \%$ CO2 incubator at $37^{\circ} \mathrm{C}$.

\section{Rabbit immunization and Poly Abs preparation}

The Poly Abs was generated by immunizing New Zealand White Rabbits with bFGF-activated fibroblasts using $1 \times$ to $5 \times 10^{7}[16,17]$ per subcutaneous injection on D0, D14, and D21. The primary inoculation was with Freund's complete adjuvant (Sigma-aldrich, USA). The serum was pooled one week later after the last immunity. Blood was allowed to clot at $4{ }^{\circ} \mathrm{C}$, and the serum was removed from the top of the mixture after centrifugation. Immunoglobulin was purified by affinity chromatography (protein A sephrose) and freeze dried. Poly Abs were kept frozen at $-80^{\circ} \mathrm{C}$ until used. Control rabbit IgG was similarly purified from whole normal rabbit serum.

\section{Enzyme-linked immunosorbent assay (ELISA)}

Tumor cells $\left(5 \times 10^{3}\right.$ per well $)$ were grown overnight in 96-well plate for ELISA assay. The media were removed and the cells were washed with PBS three times. After washing, the cells were fixed for 10 min in $70 \%$ ethanol, and blocked with $0.5 \%$ BSA for 1 $\mathrm{h}$ at room temperature. The cells were washed with PBS three times before addition of Poly Abs. The Poly Abs was diluted from 1:1,000 to $1: 10,000$ and incubated overnight at $4{ }^{\circ} \mathrm{C}$. The Poly Abs was then removed and the cells were washed with PBST (PBS containing $0.05 \%$ Tween-20) three times. The horseradish peroxidase (HRP) conjugated secondary antibody (1:5,000, BBI, China) was added to the cells and incubated for $30 \mathrm{~min}$. Finally, add $100 \mathrm{ul}$ of TMB solution (BBI, China) to each well and read absorbance at $450 \mathrm{~nm}$. Each experiment was performed in triplicate.

\section{Cell viability analysis}

Cell viability analysis was determined by 3-(4,5-dimethyl-thiazol-2-yl)-2, 5-diphenyltetrazolium bromide (MTT, Sigma-aldrich, USA) assay. Brief, 3T3, CT26, LL/2 and B16 cells were cultured $\left(5 \times 10^{3}\right.$ cells/well) in 96-well plates and incubated for $24 \mathrm{~h}$. 
The cells were treated with indicated concentrations of control rabbit IgG, or Poly Abs $(10-1000 \mu \mathrm{g} / \mathrm{ml})$ for $24 \mathrm{~h}$. The MTT assay was then performed by dissolving the reaction product in DMSO and measure the absorbance at $490 \mathrm{~nm}$.

\section{Colony formation analysis}

CT26, LL/2 and B16 cells were cultured in 6-well plates at a density of 1000 cells/well and maintained in the medium with $100 \mu \mathrm{g} / \mathrm{ml}$ Poly Abs or control IgG. After culture for 7 days, the cells were stained with $0.1 \%$ crystal violet (Beyotime, China), photographed and counted.

\section{Nuclear chromatin staining for apoptosis}

Morphologic changes in nuclei were investigated by staining cells with Hoechst 33258 (Beyotime, China). CT26, LL/2 and B16 cells were cultured on cover-glass in 6-well plates and treated with Poly Abs or control IgG $(150 \mu \mathrm{g} / \mathrm{ml})$ for $24 \mathrm{~h}$. Cells were fixed with $70 \%$ ethanol and washed with PBS. $1 \mu \mathrm{g} / \mathrm{ml}$ Hoechst 33258 was used to stain the cells for $5 \mathrm{~min}$. Finally, apoptosis was visualized with fluorescence microscopy (Olympus, Japan).

\section{Apoptosis analysis by Annexin V-FITC and PI staining}

AnnexinV-FITC and PI staining were used to detect apoptosis at an early stage according to the method of Zhang et al [17]. Briefly, the CT26, LL/2 and B16 cells $\left(2 \times 10^{5}\right)$ were cultured in 6-well plates and treated with control IgG, or Poly Abs (150 $\mathrm{ug} / \mathrm{ml})$. The cells were harvested $24 \mathrm{~h}$ latter, and washed with PBS. Cells were re-suspended at $1 \times 10^{6}$ $/ \mathrm{ml}$ in binding buffer, and the apoptotic and necrotic cells was evaluated using an Annexin V-FITC/propidium iodide (PI) apoptosis detection kit (Life Technologies, USA) according to the manufacturer's instructions.

\section{Wound healing analysis}

CT26, LL/2 and B16 cells were cultured in 6-well plates at a density of $2 \times 10^{5}$ cells/well. After $24 \mathrm{~h}$ incubation, they reached $\sim 80-90 \%$ confluence as a monolayer. A denuded area was created across the diameter of dish by a $1 \mathrm{ml}$ pipette tip. And then the cells were gently washed with the PBS to remove the detached cells. The tumor cells were treated with 120 $\mu \mathrm{g} / \mathrm{ml}$ Poly Abs or IgG for $24 \mathrm{~h}$, and the wounds were photographed. Images were analyzed with Image Pro Plus 6.0 software.

\section{Transwell analysis}

Cell migration assay was conducted to evaluate the effect of Poly Abs on the migration of CT26, LL/2 and B16 cells in vitro using a 24-well Transwell chamber $(8 \mu \mathrm{m}$, Costar, Cambridge, MA, USA). $5 \times 10^{4}$ CT2 6 cells was seeded in the insert with $200 \mu 1$ high-glucose DMEM containing $10 \%$ of FBS, and 600 $\mu$ l same complete medium was added in the lower chamber. After $24 \mathrm{~h}$ culture, the cells were treated with $120 \mu \mathrm{g} / \mathrm{ml}$ Poly Abs or control IgG for $24 \mathrm{~h}$. The cells were continuously cultured in serum free DMEM medium for $8 \mathrm{~h}$. Then the insert was placed on 24-well plate containing $600 \mu \mathrm{l}$ of high-glucose DMEM complemented with $10 \%$ FBS as a chemo-attractant. After $24 \mathrm{~h}$ culture, the cells on the upper surface of the insert were removed gently with cotton-tipped swabs and fixed with $70 \%$ ethanol for $30 \mathrm{~min}$ at room temperature. The inserts were washed with PBS three times and stained with $0.1 \%$ crystal violet for $5 \mathrm{~min}$. The number of migrating cells was counted and recorded in five randomly selected fields, and the mean number of cells was calculated.

\section{Xenograft mouse model}

CT26 cells were implanted subcutaneously into the right flanks of BALB/c mice. When the tumor nodules were palpable, the mice were divided randomly into two groups with five mice each group, and treated with control IgG or Poly Abs via the tail vein. Control IgG and Poly Abs (500 $\mu \mathrm{g} /$ dose) were administered every 3 days for 3 weeks. The tumor volume was measured with a caliper every 3 day using the formula: volume $=$ length $\times$ width $^{2} \times 0.52$.

\section{Immunohistochemistry}

To explore whether Poly Abs could effectively inhibit tumor cell proliferation in vivo, anti-Ki67 antibody (Cell Signaling, USA) was used to determine cellular proliferation in tumor tissues. Tumor tissue were fixed in $4 \%$ paraformaldehyde $48 \mathrm{~h}$ latter last treatment, embedded in paraffin, and $4 \mu \mathrm{m}$ sections were incubated with anti-Ki67 antibody (1:100) overnight at $4{ }^{\circ} \mathrm{C}$. Sections were incubated with biotin-labeled secondary antibody (1:100, Beyotime, China) for $40 \mathrm{~min}$, and then exposed to HRP-labeled streptavidin (1:200, Beyotime, China) for $20 \mathrm{~min}$. Positive reactions were visualized using 3 , 3'-diaminobenzidine tetrahydrochloride (DAB; Beyotime, China), followed by counterstaining with hematoxylin (Beyotime, China).

\section{Tail vein metastatic analysis}

For lung metastatic experiments, $500 \mu \mathrm{g} /$ dose control IgG or Poly Abs was injected into the recipient $\mathrm{BALB} / \mathrm{c}$ and $\mathrm{C} 57$ mice through the tail vein; then tumor cells were re-suspended in $100 \mu$ l PBS (CT26 and LL/2, $3 \times 10^{5} ; \mathrm{B} 16,2 \times 10^{5}$ ) and injected into $\mathrm{BALB} / \mathrm{c}$ or C57 mice via the tail vein on the same day. The treatment carried out every 3 days for 3 weeks. 
The mice were sacrificed, and the lesions were counted.

\section{Statistical analysis}

Data from the control and Poly Abs group were analyzed by a two-tailed Student's t-test. $P$ value of less than 0.05 was regarded as statistically significant.

\section{Results}

\section{Production and evaluation of Poly Abs}

Two rabbits were vaccinated with bFGF-activated fibroblasts to produce Poly Abs. ELISA analysis indicated that Poly Abs bound to CT26, LL/2, B16 and bFGF-treated fibroblasts with 10000 times dilution (Fig. 1). This result demonstrated that Poly Abs could target CT26, LL/2, B16 and bFGF-treated fibroblasts. Poly Abs bound to these cells with 10000 times dilution.

\section{Poly Abs inhibited tumor cell proliferation and colony formation}

MTT analysis was performed to assess the potential effects of Poly Abs on the CT26, B16 and LL/ 2 tumor cells viability. Tumor cells were cultured with or without Poly Abs in medium at $37^{\circ} \mathrm{C}$ for $24 \mathrm{~h}$. Cell viability was measured by a colorimetric assay utilizing MTT. Poly Abs significantly inhibited tumor cell proliferation in a dose-dependent manner when compared with the control IgG (Fig. 2A). We also found that Poly Abs did not show any inhibition in the normal 3T3 cell line (Supplementary Fig. S1). NS indicated normal saline treated group.

To confirm the results, colony formation analysis was performed to further clarify the anti-proliferative effects of Poly Abs on CT26, LL/2 and B16 cells. Tumor cells were cultured in 6-well plates and cultured with or without Poly Abs in medium for 7 days. As expected, the number and size of colonies of CT26, LL/2 and B16 cells sharply decreased in the presence of Poly Abs (Fig. 2B). These results suggested that the Poly Abs had the potent anti-proliferation effects on tumor cells. Columns, mean; bars, $\mathrm{SD}\left(\mathrm{n}=3 ;{ }^{*}, p<0.05 ;{ }^{* *}, p<0.01\right)$.

\section{Poly Abs induced tumor cell apoptosis}

A decrease in tumor cell proliferation may result from the induction of apoptosis, so we speculated that Poly Abs might induce apoptosis in CT26, LL/2 and B16 cells. To investigate this possibility, CT26, LL/2 and B16 cells were treated with $150 \mu \mathrm{g} / \mathrm{ml}$ Poly Abs or control IgG, respectively. After $24 \mathrm{~h}$ treatment, the tumor cells were stained with Hoechst 33258. The tumor cells, which were treated with Poly Abs, showed condensed and fragmented nuclei (red arrow), which are typical morphological features of apoptotic cells; while the control IgG-treated cells did not show the characteristics of apoptosis (Fig. 3A). Furthermore, quantitative assessment was further done by flow cytometric analysis. Living cell populations were gathered in the Q3 quadrant; early apoptotic cells (still viable) were in the Q4 quadrant; late apoptotic/necrotic cells (non-viable) were in the Q2 quadrant. The results indicated that the treatment with $150 \mu \mathrm{g} / \mathrm{ml}$ Poly Abs induced $32.67 \%$ LL/ 2 cells apoptosis, $25.06 \%$ CT26 cells apoptosis, and $36.97 \%$ B16 cells apoptosis (Fig. 3B). Thus, these results indicated that Poly Abs might induce CT26, LL/2 and B16 cells apoptosis.
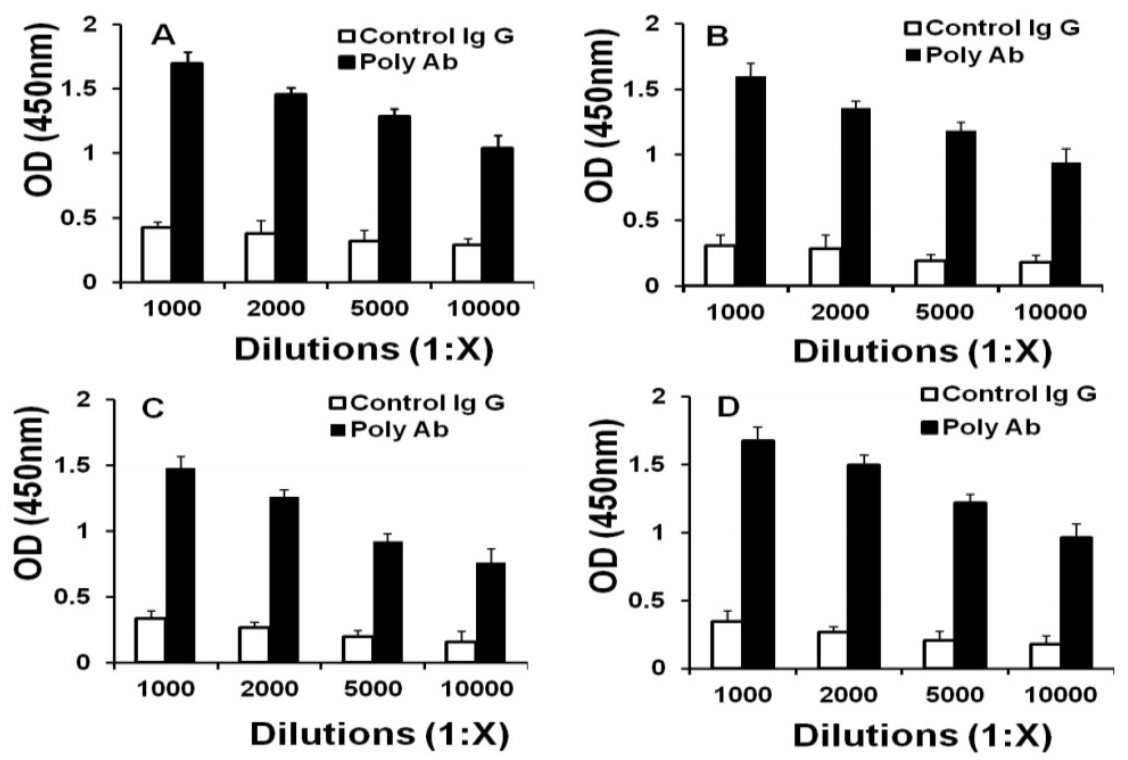

Figure 1. Binding of Poly Abs to cells. ELISA was performed to determine the proper dilution of Poly Abs against (A) B16 cells. (B) CT26 cells; (C) LL/2 cells; (D) bFGF-treated fibroblasts. 

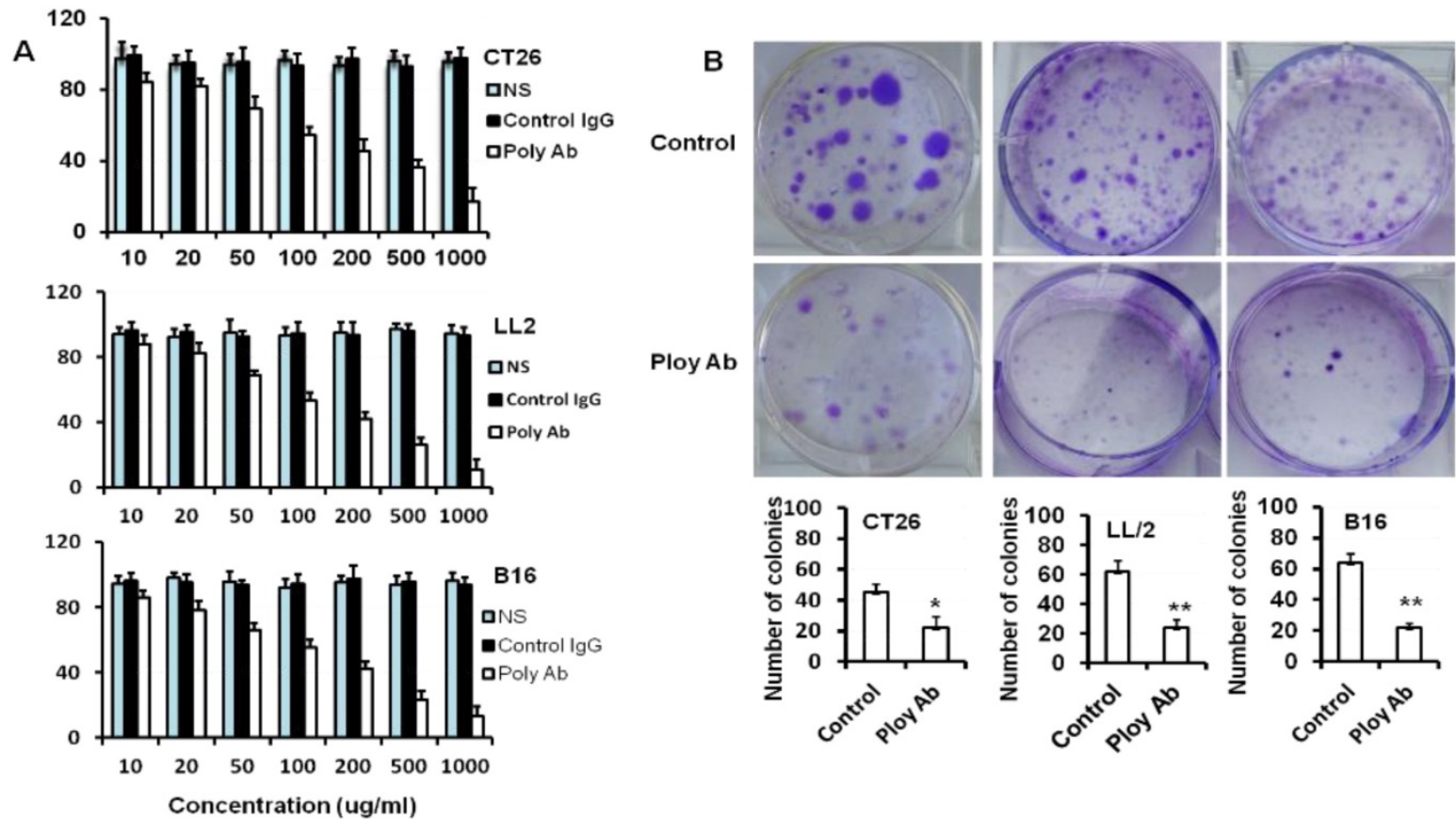

Figure 2. Poly Abs inhibited tumor cells proliferation. (A) MTT assay was used to assess the effect of Poly Abs on the CT26, B16 and LL/2 cells viability. (B) Colony formation analysis.

\section{Poly Abs inhibited the migration of tumor cells}

Enhanced migratory capacity is a particular feature of metastasizing cancer cells. To examine the anti-migration ability of Poly Abs, the wounding healing assay was performed. CT26, B16 and LL/2 tumor cells were cultured in 6-well plates and treated with $120 \mu \mathrm{g} / \mathrm{ml}$ Poly Abs or control Ig G, and photographed $24 \mathrm{~h}$ latter. The control tumor cells showed almost complete closure of wound in a confluent monolayer after $24 \mathrm{~h}$ treatment, whereas Poly Abs obviously inhibited wound closure (Fig 4A). Moreover, transwell migration assay showed that Poly Abs significantly decreased the amount of CT26 cells migration from the upper surface to the lower surface of the transwell insert (Fig. 4B). We also observed the same result on B16 and LL/2 tumor cells (Supplementary Fig. S2). The lung metastatic experiments further proved that Poly Abs significantly inhibited the metastasis of CT26 cells in vivo (Fig. 4C). The Poly Abs also significantly inhibited the lung metastasis of LL/2 and B16 tumor cells (Supplementary Fig. S3). Taken together, these data indicated that Poly Abs had significantly impact on migration and metastasis of tumor cells.

\section{Poly Abs inhibited tumor growth and proliferation in vivo}

We used CT26 tumor models to investigate the effect of Poly Abs on tumor growth and proliferation in vivo. CT26 subcutaneous xenografts were treated for 3 weeks via tail vein. Compared with the control group, administration of Poly Abs significantly inhibited the tumor growth (Fig. 5A). After mice were sacrificed, the tumor samples were taken out and applied for IHC. We performed Ki67 staining to assess the effect of Poly Abs on tumor cell proliferation. As expected, the inhibition of Ki67 expression was observed in tumor tissues treated with Poly Abs (Fig. $5 B)$.

\section{Discussion}

Increasing evidence is emerging showing that immunotherapy is a promising method to treat cancer due to the ability to selectively eliminate tumor cells. Some monoclonal antibodies (MAbs) have been already approved for cancer treatment [18], such as cetuximab and trastuzumab (Herceptin), the most advanced MAbs targeting HER1 and HER2, respectively. But only some patients are benefited, and the clinical success of these antibodies is limited with the resistant variants emerging $[19,20]$. Tumor cells often recruited redundant signaling cascades to sustain their proliferation and progression. Blocking one signal pathway, the tumor cells can regulate other signaling cascades to provide compensatory survival signals [20,21]. Hence, simultaneously blocking several epitopes could be a striking strategy to avoid resistance associated with the expression of these compensatory molecules. Bispecific antibodies (BsAbs), which simultaneously bind to 2 different antigens [22,23], are an emerging class of antibody therapeutics. Many formats of BsAbs have been engineered using recombinant approaches. Treatment of human non-Hodgkin lymphoma (NHL)-engrafted 
mice with BsAbs that target CD47 along with CD20

treated with either anti-CD47 or anti-CD20 alone [24].

significantly prolonged survival, compared to mice

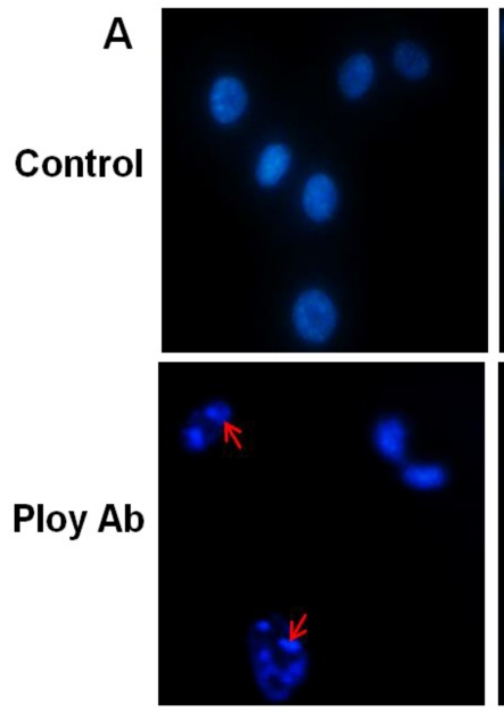

LL/2
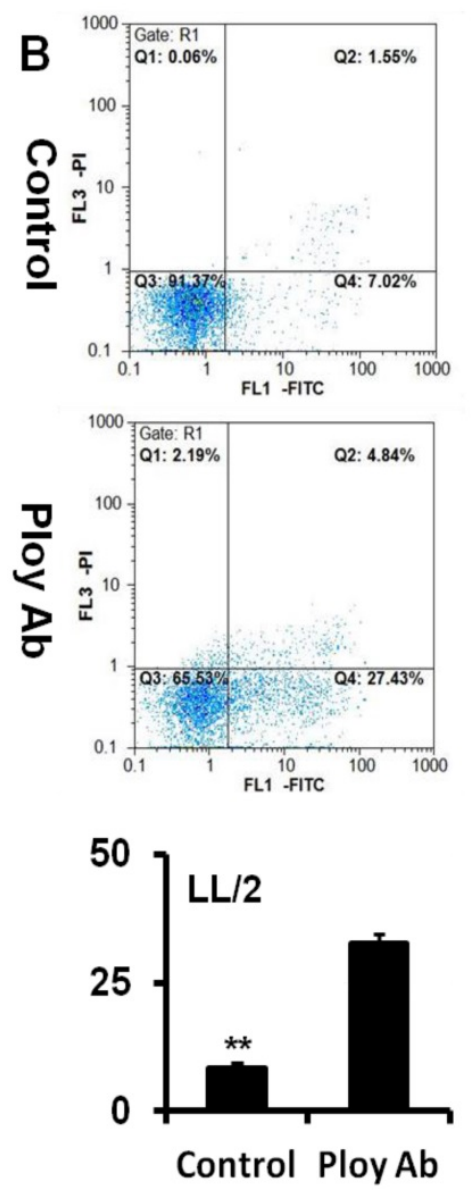
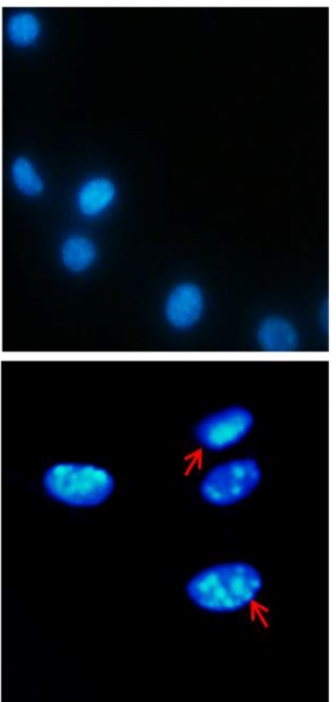

CT26
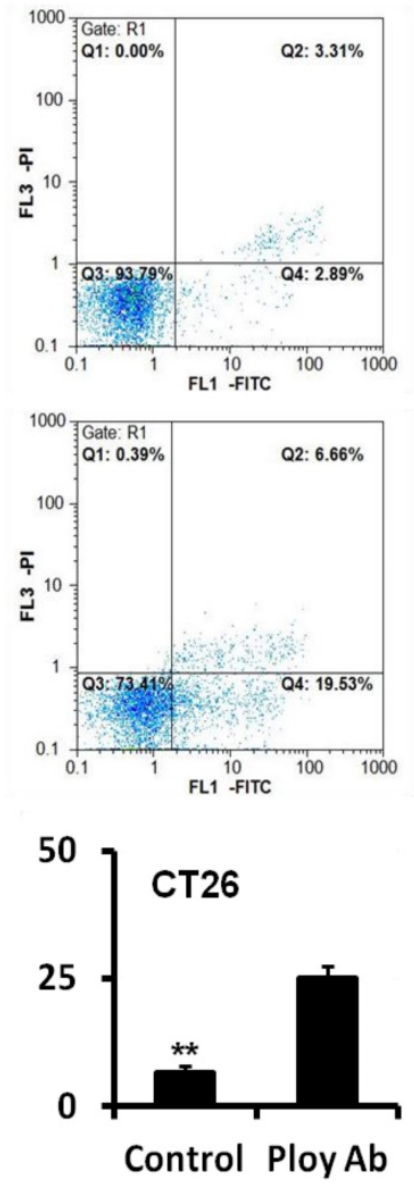

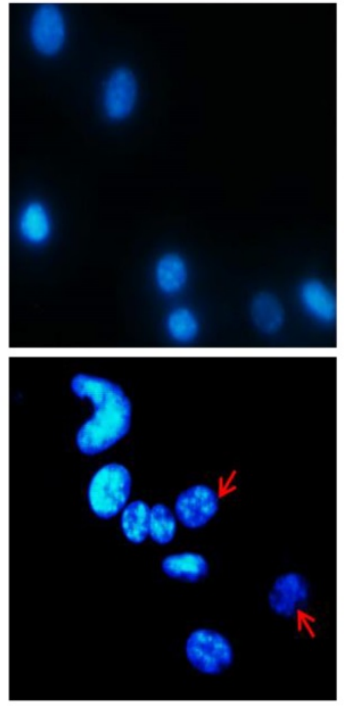

B16
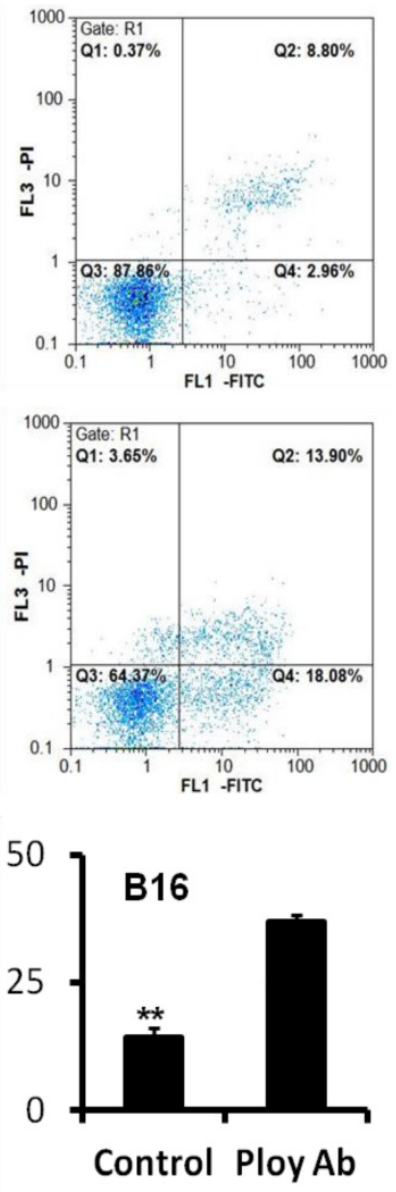

Figure 3. Poly Abs induced tumor cells apoptosis. (A) Morphologic changes in nuclei were investigated by staining cells with Hoechst 33258 ( $\times 200$ ). The red arrow indicated the condensed and fragmented nuclei. (B) Annexin V-FITC and PI staining was used to quantitatively assess apoptosis at an early stage by flow cytometric analysis. Columns, mean; bars, SD $(\mathrm{n}=3 ; *, p<0.05 ; * *, p<0.01)$ 


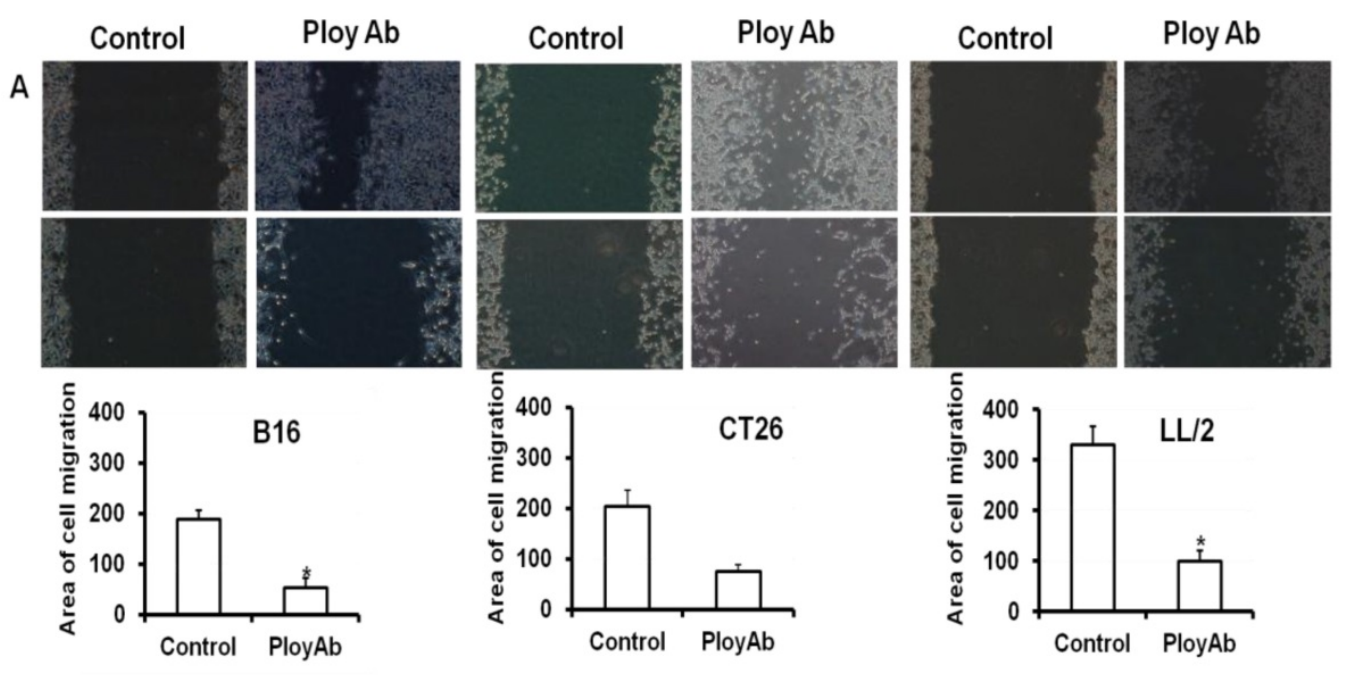

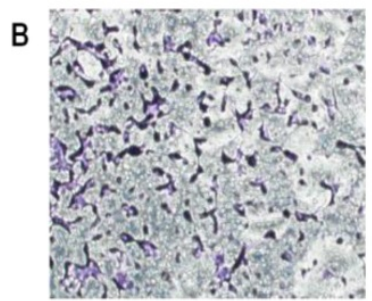

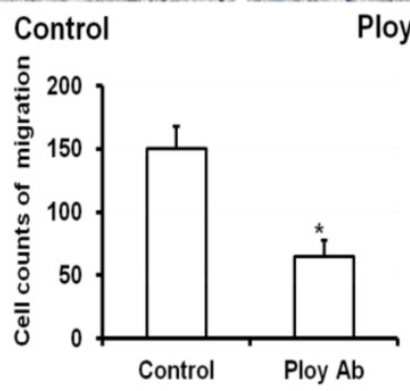

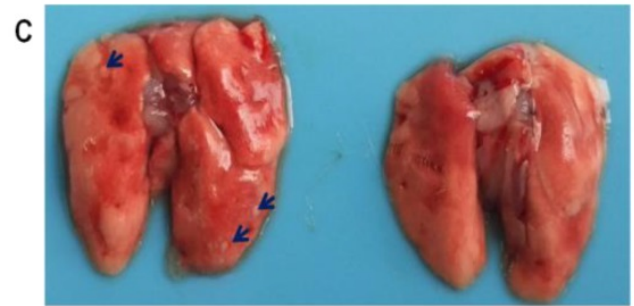

Control Ploy Ab

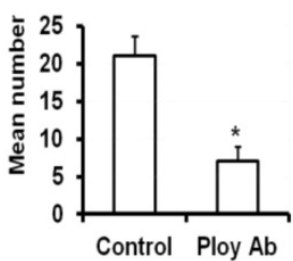

Figure 4. Poly Abs inhibited tumor cells migration and metastasis. (A) The wounding healing analysis $(x 40)$. (B) Transwell migration analysis (x 200). (C) Lung metastasis in vivo. The blue arrow indicated the tumor nodules in lung tissues. Columns, mean; bars, SD ( $=3 ; *, p<0.05)$.
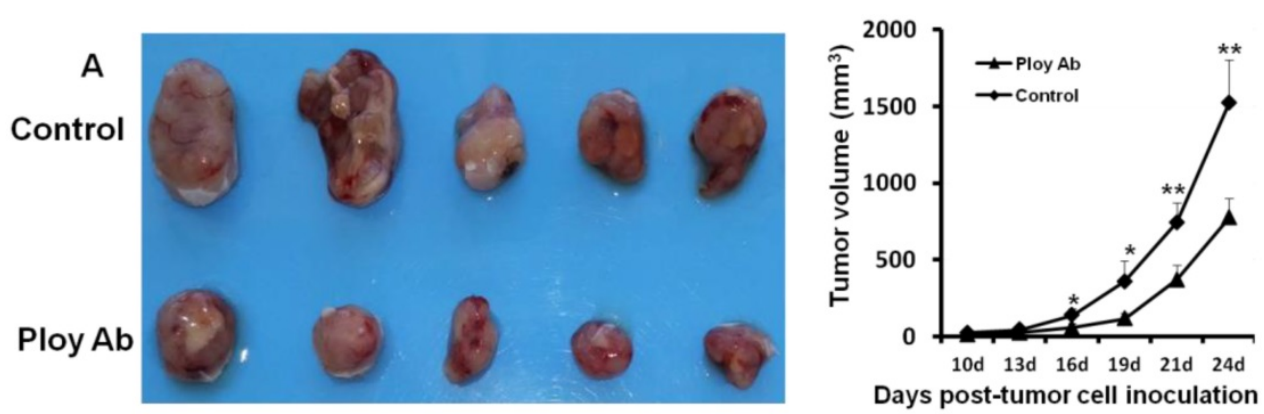

Days post-tumor cell inoculation

\section{B}

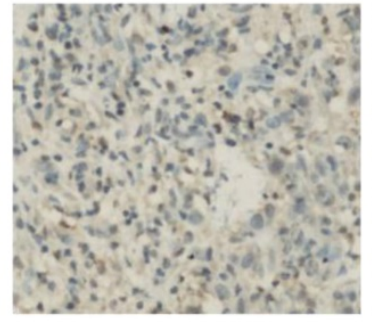

Control

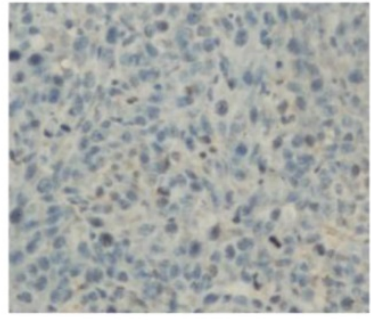

Ploy Ab

Figure 5. Poly Abs inhibited CT26 growth and proliferation in vivo. (A) Antitumor effects of Poly Abs in vivo. $(n=5, *, p<0.05 ; * *, p<0.01)$. (B) Ki67 staining was performed to assess the effect of Poly Abs on CT26 cells proliferation. 
Indeed, using Poly Abs instead of MAbs or BsAbs is the best way to trigger the anti-tumor immune response. Poly Abs can simultaneously target multiple epitopes of the same or different TAAs. Study indicated that simultaneous inactivation of HER1 and HER2 by specific Poly Abs was able to reduce the viability of a panel of human tumor lines with differential expression levels of HER1 and HER2, and circumvent the resistance mechanism [25]. There is growing recognition that the treatment effect of tumor immunotherapy is affected by many factors besides the antigenicity and/or mutational burden of cancer. Tumor microenvironment (TME) is characterized by multiple immune suppressive mechanisms, which can inhibit the effect of immune effector cells. As a result, tumor cells are able to escape the control operated by the immune system $[26,27]$. In part due to its critical role in tumor progression, metastasis and chemo-resistance, the most provocative potential targets for immunotherapy are associated with tumor microenvironment. CAFs are the main components of the stroma in most solid cancer [28]. A growing body of evidence suggested that CAFs were demonstrated to contribute to the local immunosuppressive microenvironment $[29,30]$. Targeting CAFs and the tumor microenvironment may relieve the immunosuppressive effect [31]. FAPa-based vaccines can be used to elicit FAPa-specific cytotoxic T cell (CTL) responses and elimination of the immunosuppressive environment [32,33]. FAP-specific CAR-T cells inhibit tumor growth in part by enhancing tumor-specific immune responses [34,35]. Moreover, CAFs are genetically more stable compared with the tumor cells, rendering them excellent targets for cancer immunotherapy [36,37]. Thus CAFs have become an attractive target for cancer immunotherapy.

It is widely believed that apoptosis is the process of programmed cell death that occurs under various physiological and pathological conditions. Accumulating evidence suggested that inducing cancer cell apoptosis is useful in cancer treatment. In the present study, we prepared Poly Abs by immunizing rabbits with the bFGF-activated fibroblasts. The Poly Abs inhibited the cancer cells proliferation, colony formation. Meanwhile, Poly Abs inhibited CT26 growth in the subcutaneous xenograft mouse models. The Annexin V-FITC/PI staining and nuclear chromatin staining analysis indicated that Poly Abs treatment increased tumor cells apoptosis.

The cancer cells in the carcinoma in situ were segregated from the stroma by the basement membrane $(\mathrm{BM})$. The $\mathrm{BM}$ is mainly composed of collagen IV and laminin networks produced by coordinated actions of cancer cells and stromal fibroblasts [38,39]. Cancer cells can perforate the BM using matrix metalloproteinases (MMP)-rich invadopodia [40,41]. However, CAFs are a major source of MMPs. CAFs modify the ECM by increased expression and activation of MMPs [42,43]. MMPs can interrupt tissue polarity and architecture, as well as enhance the abilities of cancer cells to inhibit E-cadherin-mediated adhesion and navigate the stromal ECM constrains [44]. In addition, CAFs secrete ECM proteins, such as fibronectin and a variety of collagen, which were necessary for invasion of cancer cells [45]. Producing ECM by CAFs allows invasion and metastasis, so we speculated that the Poly Abs could inhibit migration and metastasis. As expected, our study indicated that Poly Abs could inhibit tumor cell migration and metastasis (Figure 4, Supplementary Figures S2 and 3).

When the immunogen is living cells, the polyclonality of the antibody response helps guard against the development of cell "escape variants" [15]. In this study, we used the living bFGF-activated fibroblasts as immunogen. The advantage is that all the molecules on the CAFs cells, including unknown molecules, are exposed to the immune system. Our data supported further exploring the CAFs-targeting vaccines for active immunotherapy of cancer. There are still several limitations in our study. For example, the antigens recognized by Poly Abs are unclear at present, and their characterization is now in progress.

Taken together, our results indicated that Poly Abs could decrease tumor growth and promote tumor cells apoptosis; the Poly Abs can inhibit the migration and metastasis of tumor cell. Poly Abs and its derivates are promising candidates for cancer immunotherapy.

\section{Supplementary Material}

Supplementary figures.

http://www.ijbs.com/v14p1621s1.pdf

\section{Acknowledgements}

The authors gratefully acknowledge the National Natural Science Foundation of China (No. 81560491) for the financial support.

\section{Competing Interests}

The authors have declared that no competing interest exists.

\section{References}

1. Belli C, Trapani D, Viale G, et al. Targeting the microenvironment in solid tumors. Cancer Treat Rev. 2018; 65: 22-32.

2. Hanahan D, Weinberg RA. Hallmarks of cancer: the next generation. Cell. 2011; 144: 646-74.

3. Postow MA, Callahan MK, Wolchok JD. Immune checkpoint blockade in cancer therapy. J Clin Oncol. 2015; 33:1974-1982. 
4. Kontermann RE, Brinkmann U. Bispecifc antibodies. Drug Discov Today. 2015; 20: 838-847.

5. Guo $Y$, Wang $Y$, Han W. Chimeric antigen receptor-modifed T cells for solid tumors: challenges and prospects. J Immunol Res. 2016; 3850839

6. Kalluri R. The biology and function of fibroblasts in cancer. Nature Reviews Cancer. 2016; 16: 582-598

7. Yeung TL, Leung CS, Li F, et al. Targeting stromal-cancer cell crosstalk networks in ovarian cancer treatment. Biomolecules. 2016; 6: 3 .

8. Yeung TL, Leung CS, Wong KK, et al. TGF- $\beta$ modulates ovarian cancer invasion by upregulating CAF-derived versican in the tumor microenvironment. Cancer Res. 2013; 73: 5016-5028.

9. Su S, Chen J, Yao H, et al. CD10+GPR77+ Cancer-Associated Fibroblasts Promote Cancer Formation and Chemoresistance by Sustaining Cancer Stemness. Cell. 2018; 172: 841-856.

10. Shimoda M, Principe S, Jackson HW, et al. Loss of the Timp gene family is sufficient for the acquisition of the CAF-like cell state. Nat Cell Biol. 2014; 16: 889-901.

11. Li X, Wang $Y$, Zhao $Y$, et al. Immunotherapy of tumor with vaccine based on basic fibroblast growth factor-activated fibroblasts. J Cancer Res Clin Oncol. 2014; 140: 271-280.

12. Weiner LM, Murray JC, Shuptrine CW. Antibody-based immunotherapy of cancer. Cell 2012; 148: 1081-4.

13. Cane PA. Molecular epidemiology of respiratory syncytial virus. Rev Med Virol. 2001; 11: 103-116.

14. Sullender WM. Respiratory syncytial virus genetic and antigenic diversity. Clin Microbiol Rev. 2000; 13: 1-15.

15. Sharon, J. Liebman MA, Williams BR. Recombinant polyclonal antibodies for cancer therapy. J Cell Biochem. 2005; 96: 305-313.

16. Mu B, Yang JL, Gou LT, et al. Polyclonal rabbit anti-murine plasmacytoma cell globulins induce myeloma cells apoptosis and inhibit tumour growth in mice. Apoptosis. 2011; 16: 370-81.

17. Zhang S, Yu M, Deng H, et al. Polyclonal rabbit anti-human ovarian cancer globulins inhibit tumor growth through apoptosis involving the caspase signaling. Sci Rep. 2014; 4: 4984.

18. Costa A, Kieffer Y, Scholer-Dahirel A, et al. Fibroblast heterogeneity and immunosuppressive environment in human breast cancer. Cancer Cell. 2018; 33: 1-17.

19. Carmeliet P, Jain RK. Principles and mechanisms of vessel normalization for cancer and other angiogenic diseases. Nat Rev Drug Discov. 2011; 10: 417-427.

20. Fukumura D, Duda DG, Munn LL, et al. Tumor microvasculature and microenvironment: novel insights through intravital imaging in preclinical models. Microcirculation. 2010; 17: 206-225.

21. Schlom J. Therapeutic cancer vaccines: current status and moving forward. J Natl Cancer Inst. 2012; 104: 599-613.

22. Velasquez MP, Bonifant CL, Gottschalk S. Redirecting T cells to hematological malignancies with bispecific antibodies. Blood. 2018; 131: 30-38.

23. Pereira LMS, Gomes STM, Ishak R, et al. Regulatory T cell and forkhead box protein 3 as modulators of immune homeostasis. Front Immunol. 2017; 8: 605.

24. Piccione EC, Juarez S, Liu J, et al. A bispecific antibody targeting CD47 and CD20 selectively binds and eliminates dual antigen expressing lymphoma cells. MAbs. 2015; 7: 946-56.

25. Suárez NG, Báez GB, Rodríguez MC, et al. Anti-proliferative and pro-apoptotic effects induced by simultaneous inactivation of HER1 and HER2 through endogenous polyclonal antibodies. Oncotarget, 2017; 8: 82872-82884.

26. Pulluri B, Kumar A, Shaheen M, et al. Tumor microenvironment changes leading to resistance of immune checkpoint inhibitors in metastatic melanoma and strategies to overcome resistance. Pharmacol Res. 2017; 123: 95-102.

27. Munn DH, Bronte V. Immune suppressive mechanisms in the tumor microenvironment. Curr Opin Immunol. 2016; 39: 1-6.

28. Santi A, Kugeratski FG, Zanivan S. Cancer Associated Fibroblasts: The Architects of Stroma Remodeling. Proteomics. 2017: 18: 1700167.

29. Takahashi H, Sakakura K, Kudo T, et al. Cancer-associated fibroblasts promote an immunosuppressive microenvironment through the induction and accumulation of protumoral macrophages. Oncotarget. 2017; 8: 8633-8647.

30. Becker JC, Andersen MH, Schrama D, et al. Immune-suppressive properties of the tumor microenvironment. Cancer Immunol. Immunother. 2013; 62: 1137-48

31. Deng Y, Cheng J, Fu B, et al. Hepatic carcinoma-associated fibroblasts enhance immune suppression by facilitating the generation of myeloid-derived suppressor cells. Oncogene. 2017; 36: 1090-1101.

32. Kraman M, Bambrough PJ, Arnold JN, et al. Suppression of antitumor immunity by stromal cells expressing fibroblast activation protein- $a$. Science. 2010; 330: 827-830

33. Fearon DT. The carcinoma-associated fibroblast expressing fibroblast activation protein and escape from immune surveillance. Cancer Immunol Res. 2014; 2: 187-193.

34. Wang L-CS, Lo A, Scholler J, et al. Targeting fibroblast activation protein in tumor stroma with chimeric antigen receptor T cells can inhibit tumor growth and augment host immunity without severe toxicity. Cancer Immunol Res 2014; 2: 154-66.

35. Lo A, Wang LS, Scholler J, et al. Tumor-Promoting Desmoplasia Is Disrupted by Depleting FAP-Expressing Stromal Cells. Cancer Res. 2015; 75: 2800-2810.
36. Ishiguro $\mathrm{K}$, Yoshida $\mathrm{T}$, Yagishita $\mathrm{H}$, et al. Epithelial and stromal genetic instability contributes to genesis of colorectal adenomas. Gut. 2006; 55: 695-702.

37. Jiang H, Hegde S, DeNardo DG. Tumor-associated fibrosis as a regulator of tumor immunity and response to immunotherapy. Cancer Immunol Immunother. 2017; 66: 1037-1048.

38. Glentis A, Gurchenkov V, Matic Vignjevic D. Assembly, heterogeneity, and breaching of the basement membranes. Cell Adh Migr. 2014; 8: 236-245.

39. LeBleu VS, Macdonald B, Kalluri R. Structure and function of basement membranes. Exp Biol Med. 2007; 232: 1121-1129.

40. Hotary KB, Yana I, Sabeh F, et al. Matrix metalloproteinases (MMPs) regulate fibrin-invasive activity via MT1-MMP-dependent and -independent processes. J Exp Med. 2002; 195: 295-308.

41. Linder S, Wiesner C, Himmel M. Degrading devices: invadosomes in proteolytic cell invasion. Annu Rev Cell Dev Biol. 2011; 27: 185-211.

42. Miles FL, Sikes RA. Insidious changes in stromal matrix fuel cancer progression. Mol Cancer Res. 2014; 12: 297-312.

43. Tomasek JJ, Gabbiani G, Hinz B, et al. Myofibroblasts and mechano-regulation of connective tissue remodelling. Nat Rev Mol Cell Biol. 2002; 3: 349-63.

44. Erdogan B, Webb DJ. Cancer-associated fibroblasts modulate growth factor signaling and extracellular matrix remodeling to regulate tumor metastasis. Biochem Soc Trans. 2017; 45: 229-236.

45. De Wever O, Nguyen QD, Van Hoorde L, et al. Tenascin-C and SF/HGF produced by myofibroblasts in vitro provide convergent pro-invasive signals to human colon cancer cells through RhoA and Rac. FASEB J. 2004; 18: 1016-1018 